\title{
Rigorous Properties of a Continuous System in the High Activity Region
}

\author{
M. Cassandro \\ Istituto di Fisica dell'Università di Roma - Italy \\ Istituto Nazionale di Fisica Nucleare - Sezione di Roma \\ A. Da Fano \\ Istituto di Matematica dell'Università di Roma - Italy \\ C.N.R., Gruppo Nazionale Fisica Matematica \\ Received December 18, 1973

\begin{abstract}
The original idea of Ruelle, to associate classes of configurations of the continuous Widom and Rowlinson model to contours on a lattice, is exploited to get for this model an expression of the grand partition function very similar to that of the Ising system. This allows to get, when the activities are high enough, a microscopic description of this system as a mere transcription of Minlos and Sinai analysis of the Ising model at low temperatures.
\end{abstract}

\section{Introduction}

In the past decade Minlos and Sinai [1,2], using the original idea due to Peierls [3] of contours on a lattice were able to derive a set of integral equations à la Kirkwood and Saltzburg for the "contour correlation functions" and get a very detailed microscopic description for a large class of lattice systems of the Ising type when the temperature is sufficiently low.

The aim of this paper is to show how it is possible to extend the methods of Minlos and Sinai and their results to the case of a continuous system: the Widom and Rowlinson model. Such a model [4] is a classical continuous system with two kinds of particles, $\mathrm{A}$ and $\mathrm{B}$, in which there is a hard-core repulsion of range $\mathrm{R}$ between unlike particles and no interaction between like particles.

Recently Ruelle [5], associating classes of (A-B)-particle configurations to sets of closed contours on a lattice, was able to prove the existence of a phase transition, when the activities of the two species of particles are equal and high enough.

Starting from the same geometrical description, we get, with a suitable definition of subensembles of contours (the chains), an ex- 
pression for the partition function that is very similar, in structure, to that of the Ising model and easily derive a system of integral equations for the "chain correlation functions", (see Appendix A).

Furthermore we prove that the "chain correlation functions" are bounded by a suitable decreasing function of the length of the chains perimeters, (see Appendix B).

The set of integral equations, inplemented by the above-mentioned bounds, is sufficient to fully translate the analysis, given by Minlos and Sinai for the Ising system, to the Widom and Rowlinson model. The rule is to substitute the words "low enough temperatures" by "activities equal for both species of particles and high enough".

\section{Section I}

Following Ruelle [5], we assume that the box $\Lambda$, containing the particles, is a rectangle composed of $N$ little squares with side $d=\frac{R}{3 \sqrt{2}}$, so that $R$ is the diagonal of a $3 d \times 3 d$ square.

We further assume that the space outside $\Lambda$ is completely filled by $A$ particles, so that the strip, composed of the first four squares adjacent to the boundary of $\Lambda$, cannot contain $B$-particles ( $A$-boundary conditions).

If we consider now an arbitrary configuration in $\Lambda$ (consistent with the above-mentioned boundary conditions), and shade all $3 d \times 3 d$ squares, centered on the little squares, containing at least one $B$-particle, the boundary of the union of the shaded areas will be a polygonal of various edge self-avoiding closed contours ${ }^{1}$, (see Fig. 1). Among all these contours we will consider only the subset of the outer ones (i.e. the contours that may be connected to the boundary of $\Lambda$ by a lattice path not intersecting any other contour). We will label them by $\gamma$ and define a chain $C$ as the smallest set of outer contours such that, if two outer contours have a distance less than $\frac{8 R}{3 \sqrt{2}}$, they belong to the same chain. (Notice that our definition concerns external contours only, and therefore is different from the corresponding one in Ruelle [5]).

We further say that two chains are compatible if they can be found, in the same configuration, as disjoint chains.

Given a chain $C \equiv\left(\gamma_{1}, \ldots, \gamma_{s}\right)$ we will call $\Theta(C)$ the generally disconnected region of $\Lambda$ that has $C$ as boundary. $\tilde{\Theta}(C)$ will be the region obtained by adding to $\Theta(C)$ a strip that contains all the squares at a

1 For a non ambiguous definition of edge self-avoiding contours see for instance Gallavotti, Martin Löf, Miracle-Solé in Battelle Seattle 1971 Rencontres pag. 164. BerlinHeidelberg-New York: Springer 1971. 


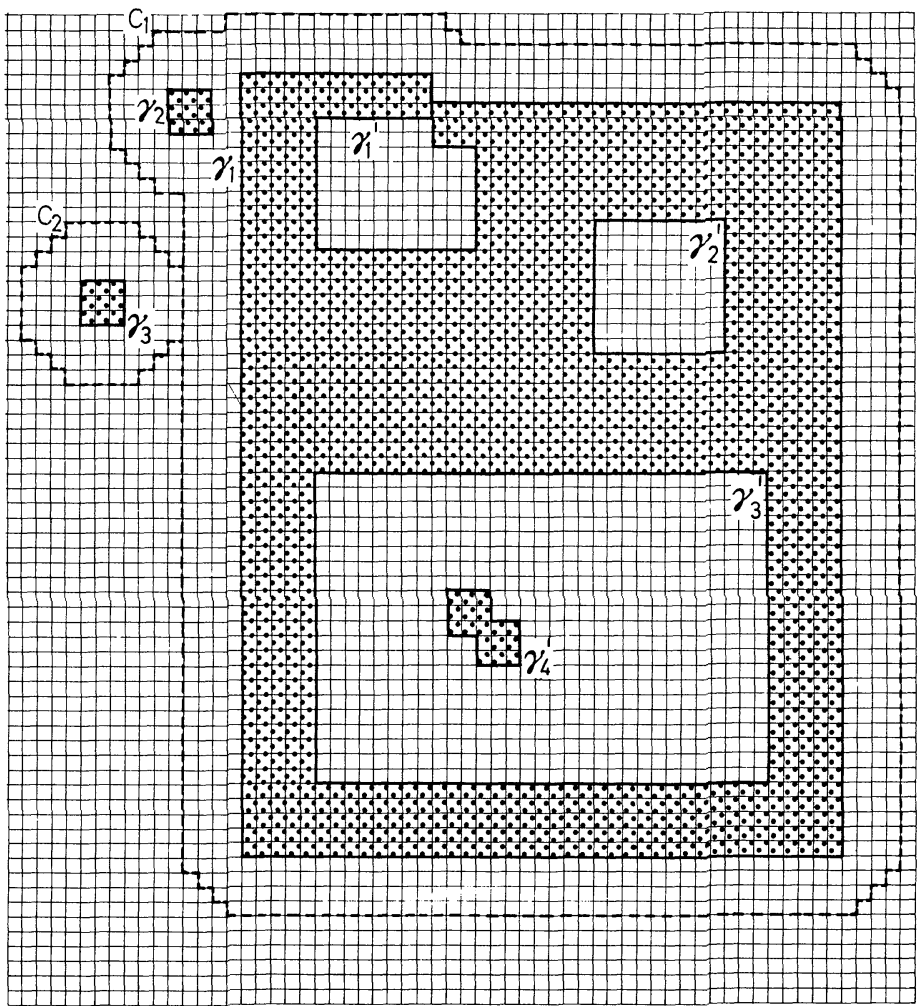

Fig. 1. The figure describes a class of $(A-B)$-particle configurations where: 1) The outer contours are $\gamma_{1}, \gamma_{2}, \gamma_{3}$. 2) The internal contours are $\gamma_{1}^{\prime}, \gamma_{2}^{\prime}, \gamma_{3}^{\prime}, \gamma_{4}^{\prime}$. 3) $\gamma_{1}$ and $\gamma_{2}$ belong to the same chain: $C_{1} ; \gamma_{3}$ is the chain $C_{2}$. 4) Following appendix B: $\gamma_{1}, \gamma_{2}, \gamma_{1}^{\prime}, \gamma_{3}^{\prime}$ belong to $\Gamma_{C}^{0}$

distance less than $R$ from the little squares, containing $B$ particles, internal to the outer contours belonging to $C$, (see Fig. 1). For convenience we shall assume that the region $\tilde{\Theta}(C)$ does not contain its boundary.

If $C$ and $C^{\prime}$ are compatible chains, $\tilde{\Theta}(C)$ and $\tilde{\Theta}\left(C^{\prime}\right)$ will not overlap, because the minimal distance between two contours belonging respectively to $C$ and $C^{\prime}$ is at least $\frac{8 R}{3 \sqrt{2}}$.

Calling $X$ an arbitrary $A-B$ configuration, consistent with $A$-particle boundary conditions, and $\mathscr{M}_{A}(\Lambda)$ the collections of all these configurations, any $X$ is associated to a well-definite set of compatible chains on the lattice and for $X$ varying in $\mathscr{M}_{A}(\Lambda)$, we get the collections of all sets of compatible chains contained in $\Lambda$. 
According to these definitions, calling $\mathscr{M}(\tilde{\Theta}(C))$ the subset of all distributions of $A-B$ particles internal to $\tilde{\Theta}(C)$ and consistent with the existence of $C$, it is easy to check that if $C$ and $C^{\prime}$ are compatible chains, the distributions belonging to $\mathscr{M}(\tilde{\Theta}(C))$ and $\mathscr{M}\left(\tilde{\Theta}\left(C^{\prime}\right)\right)$ are completely indipendent.

As a consequence of this fact, the grand partition function for the Widom and Rowlinson system, contained in the volume $\Lambda$, can be written in the following form:

$$
\Xi(\Lambda)=\sum_{\left\{C_{1}, \ldots, C_{s}\right\}}^{\prime} \prod_{1}^{s} \Xi\left(\tilde{\Theta}\left(C_{i}\right)\right) \Xi_{0}\left(\Lambda / \cup_{i} \tilde{\Theta}\left(C_{i}\right)\right)
$$

where the primed sum is over all sets of compatible chains contained in $\Lambda ; \Xi\left(\tilde{\Theta}\left(C_{i}\right)\right)$ is the grand partition function for all configurations in $\mathscr{M}\left(\tilde{\Theta}\left(C_{i}\right)\right) ; \Xi_{0}\left(\Lambda / \cup_{i} \tilde{\Theta}\left(C_{i}\right)\right)$ is the grand partition function for a gas of $A$-particles in the region $\Lambda / \cup_{i} \tilde{\Theta}\left(C_{i}\right)$.

$$
\begin{gathered}
\text { Now, from } \Xi_{0}\left(\Lambda / \cup_{i} \tilde{\Theta}\left(C_{i}\right)\right)=\frac{\Xi_{0}(\Lambda)}{\prod_{i} \Xi_{0}\left(\tilde{\Theta}\left(C_{\imath}\right)\right)} \text { we obtain } \\
\Xi(\Lambda)=\sum_{\left\{C_{1}, \ldots, C_{s}\right\}}^{\sum_{1}^{\prime}} \prod_{i}^{s} \frac{\Xi\left(\tilde{\Theta}\left(C_{i}\right)\right)}{\Xi_{0}\left(\tilde{\Theta}\left(C_{i}\right)\right)} \Xi_{0}(\Lambda) .
\end{gathered}
$$

This expression for the grand partition function has the same structure as that of the Ising model (i.e. is a sum of factorized indipendent contributions) and we can define the "chain correlation functions" (i.e. the probabilities of finding a certain finite set of compatible chains present in a configuration) by:

$$
\varrho_{m}\left(C_{1}, \ldots, C_{m} \mid \Lambda\right)=[\Xi(\Lambda)]^{-1} \sum_{\left\{C_{1}^{0}, \ldots, C_{s}^{0}\right\}}^{\prime} \prod_{1}^{s} \frac{\Xi\left(\tilde{\Theta}\left(C_{i}^{0}\right)\right)}{\Xi_{0}\left(\tilde{\Theta}\left(C_{i}^{0}\right)\right)} \Xi_{0}(\Lambda)
$$

where $\sum_{\left\{C_{1}^{0}, \ldots, C_{s}\right\}}^{\prime}$ extend over the collections of compatible chains containing $C_{1}, \ldots, C_{m}$

Following Minlos and Sinai we can write a set of equations for such functions:

$$
\begin{aligned}
& \varrho_{m}\left(C_{1}, \ldots, C_{m} \mid \Lambda\right)= \lambda\left(C_{1}\right)\left[\varrho_{m-1}\left(C_{2}, \ldots, C_{m} \mid \Lambda\right)\right. \\
&+\sum_{1}^{\infty}(-)^{k} \sum_{\left\{C_{i_{1}}^{\prime}, \ldots, C_{i_{k}}^{\prime}\right\}} \varrho_{m-1+k}\left(C_{2}, \ldots, C_{m}, C_{i_{1}}^{\prime}, \ldots, C_{i_{k}}^{\prime} \mid \Lambda\right) \\
&\left.-\sum_{l} \varrho_{m}\left(C_{2}, \ldots, C_{m}, \tilde{C}_{l} \mid \Lambda\right)\right] \\
& \varrho_{1}\left(C_{1} \mid \Lambda\right)=\lambda\left(C_{1}\right)\left[1+\sum_{1}^{\infty}(-)^{k} \sum_{\left\{C_{i_{1}}^{\prime}, \ldots, C_{t_{k}}^{\prime}\right\}} \varrho_{k}\left(C_{i_{1}}^{\prime}, \ldots, C_{i_{k}}^{\prime} \mid \Lambda\right)-\sum_{l} \varrho_{l}\left(\tilde{C}_{l} \mid \Lambda\right)\right]
\end{aligned}
$$


where the sums are over all collections of chains each of them "intersecting" ${ }^{2} C_{1}$, and over all chains "embracing" ${ }^{3} C_{1}$, and

$$
\lambda\left(C_{1}\right)=\frac{\Xi\left(\tilde{\Theta}\left(C_{1}\right)\right)}{\bar{\Xi}\left(\tilde{\Theta}\left(C_{1}\right)\right)}
$$

The quantity $\Xi\left(\tilde{\Theta}\left(C_{1}\right)\right)$, in the numerator, is the grand partition function with respect to all distributions in $\mathscr{M}\left(\tilde{\Theta}\left(C_{1}\right)\right)$ (i.e. all $(A-B)$ particle distributions in $\hat{\Theta}\left(C_{1}\right)$ associated to the chain $\left.C_{1}\right)$. On the contrary $\bar{\Xi}\left(\tilde{\Theta}\left(C_{1}\right)\right)$, in the denominator, is the grand partition function over the set $\bar{M}\left(\Theta\left(C_{1}\right)\right)$ of all $(A-B)$-particle distributions internal to $\tilde{\Theta}\left(C_{1}\right)$, that give rise to sets of chains non intersecting $C_{1}$.

The derivation of (4) and (5) is given in Appendix A. While in Appendix $\mathrm{B}$ we show that the following two inequalities hold

$$
\begin{array}{r}
\lambda\left(C_{1}\right) \leqq e^{-\alpha\left|C_{1}\right|} \\
\varrho_{m}\left(C_{1}, \ldots, C_{m} \mid \Lambda\right) \leqq e^{-\alpha \Sigma_{1}\left|C_{i}\right|}
\end{array}
$$

where $|C|$ is the total length of outer contours belonging to $C ; \alpha=\frac{z R^{2}}{72}$. Once inequalities (7) are granted, the procedure in dealing with these equations is almost standard: introduced by Ruelle for a gas system in the low activity region [6], and extended to a "gas of contours" by Minlos and Sinai [7], it is applicable to a "gas of chains of contours" with minor obvious changes [8].

The main results can be summarized in the following way. When the activities of the particles are

$$
z_{A}=z_{B}=z \geqq 144 \frac{\mathscr{R}}{R^{2}} \ln 2+\frac{72}{R^{2}}\left(\frac{\mathscr{R}+6}{6}\right) \ln 3,
$$

where $\mathscr{R}$ is equal to $\frac{8 R}{3 d \sqrt{2}}$, and $\xi=3^{\left(\frac{\mathscr{R}}{6}+1\right)} e^{-\alpha}$ the following results hold:

a) If the "chain correlation functions" $\varrho_{m}\left(C_{1}, \ldots, C_{m} \mid \Lambda\right)$ are defined by (4) and (5) there exist "infinite volume chain correlation functions"

${ }^{2} C^{\prime}$ is a chain "intersecting" $C$, if some outer contour belonging to $C^{\prime}$ "intersects" an outer one of $C$, or lies closer than $\frac{8 R}{3 \sqrt{2}}$ to it. Notice that $C$ itself is among the "intersecting" chains.

${ }^{3} \tilde{C}$ is a chain "embracing" $C$, if all the contours of $C$ are contained inside an outer contour of $\tilde{C}$ and lie at a distance larger than $\frac{8 R}{3 \sqrt{2}}$ from it. 
$\varrho_{m}\left(C_{1}, \ldots, C_{m} \mid \Lambda\right)$ such that

$$
\left|\varrho_{m}\left(C_{1}, \ldots, C_{m} \mid \Lambda\right)-\varrho_{m}\left(C_{1}, \ldots, C_{m}\right)\right| \leqq B e^{-\alpha \sum_{1}\left|C_{\imath}\right|} \xi^{\frac{2 \delta}{\mathscr{R}}}
$$

where $C_{1}, \ldots, C_{m}$ are compatible chains in $\Lambda, \delta$ is the distance of the outer contours belonging to $C_{1}, \ldots, C_{m}$ from the boundary of $\Lambda$, and $\left|C_{i}\right|$ is the total length of outer contours belonging to $C_{i} ; B$ is a constant.

b) The "chain correlation functions" and the "infinite chain correlation functions" have one and only one solution.

c) The "chain correlation functions" have the cluster property:

$$
\begin{gathered}
\left|\varrho_{m}\left(C_{1}, \ldots, C_{k}, C_{k+1}, \ldots, C_{m}\right)-\varrho_{k}\left(C_{1}, \ldots, C_{k}\right) \varrho_{m-k}\left(C_{k+1}, \ldots, C_{m}\right)\right| \\
\leqq K \xi^{\tau} e^{-\alpha \sum_{1}\left|C_{t}\right|}
\end{gathered}
$$

where $K$ is some constant and $\tau$ denotes the distance between the set of chains $\left(C_{1}, \ldots, C_{k}\right)$ and $\left(C_{k+1}, \ldots, C_{m}\right)$.

\section{Conclusions}

The physical relevance of the results of the previous section can be illustrated by the following theorem:

Theorem. if $z_{A}=z_{B}=z$ is large enough, there exist positive numbers

$$
\varrho(C) \leqq e^{-\alpha|C|}
$$

such that an (A-B)-particle configuration $X$, randomly chosen out of the ensemble $\mathscr{M}_{A}(\Lambda)$, will contain, with a probability approaching 1 as $\Lambda \rightarrow \infty$, a number $K_{(C)}(X)$ of chains congruent to $C$, such that

$$
\left|K_{(C)}(X)-\varrho(C) \frac{\operatorname{area}(\Lambda)}{d^{2}}\right| \leqq D(z) \frac{\sqrt{\operatorname{area}(\Lambda)}}{d} e^{-\alpha|C|}
$$

where $D(z)$ is a function of $z$ independent from $\Lambda$ and the relation has to be interpreted as holding simultaneously for all C's.

The proof of this theorem, once inequalities (7) are assumed, runs exactly as in the Minlos and Sinai papers $[1,2]$ to which we refer for details.

The above theorem implies that very few chains are present and in particular no chain can have a perimeter $|C|>D(z) \ln \frac{\operatorname{area}(\Lambda)}{d}$. Hence a typical $(A-B)$-particle configuration, in the grand canonical ensemble with $A$-particle boundary conditions, is such that only few small regions 
containing $B$-particles are randomly distributed in a free gas of $A$ particles only.

Furthermore, the results of this paper, in particular the cluster properties of the "chain correlation functions", together with the F.K.G. inequalities for the Widom and Rowlinson system [9] can give, in analogy to what Martin-Löf has done for the Ising system [10], an insight into other interesting properties as the central-limit theorem, for the energy and the density, and the infinite differentiability of the free energy [11].

Acknowledgements. We are greatly indebted to G. Gallavotti for helpfull suggestions and criticism.

\section{Appendix A}

In this appendix we shall derive equations for the "chain correlation functions". The procedure is that of Minlos and Sinai [7] with some obvious changes.

Let $\mathfrak{B}\left(\left\{C_{1}, \ldots, C_{m}\right\}\right)$ be the collection of chain configurations in $A$, that contain the chains $C_{1}, \ldots, C_{m}$, and let $\mathfrak{B}_{C_{1}}\left(\left\{C_{2}, \ldots, C_{m}\right\}\right)$ be the subset of elements of $\mathfrak{B}\left(\left\{C_{2}, \ldots, C_{m}\right\}\right)$ such that $C_{2}, \ldots, C_{m}$ and the remaining chains do not "intersect" and do not "embrace" $C_{1}$. (Notice that among the "intersecting" chains there is $C_{1}$ itself.)

Let now $C^{\prime}$ and $\tilde{C}$, respectively label chains "intersecting" and "embracing" $C_{1}$. From the previous definitions the likelihood

$$
\mathfrak{B}_{C_{1}}\left(\left\{C_{2}, \ldots, C_{m}\right\}\right)
$$

can be written as follows:

$$
\begin{aligned}
\mathfrak{B}_{C_{1}}\left(\left\{C_{2}, \ldots, C_{m}\right\}\right)= & \mathfrak{B}\left(\left\{C_{2}, \ldots, C_{m}\right\}\right)-\cup_{i} \mathfrak{B}\left(\left\{C_{i}^{\prime}, C_{2}, \ldots, C_{m}\right\}\right) \\
& -\cup_{l} \mathfrak{B}\left(\left\{\tilde{C}_{l}, C_{2}, \ldots, . C_{m}\right\}\right) .
\end{aligned}
$$

Then, since the $C_{i}^{\prime}$ are not mutually incompatible, the related probabilities [12] are equal to

$$
\begin{aligned}
\varrho_{C_{1}}\left(C_{2}, \ldots, C_{m} \mid \Lambda\right)= & \varrho_{m-1}\left(C_{2}, \ldots, C_{m} \mid \Lambda\right) \\
& +\sum_{1}^{\infty}(-)^{k} \sum_{\left\{C_{i_{1}}^{\prime}, \ldots C_{i_{k}}^{\prime}\right\}} \varrho_{m-1+k}\left(C_{2}, \ldots, C_{m}, C_{i_{1}}^{\prime}, \ldots, C_{i_{k}}^{\prime} \mid \Lambda\right) \\
& -\sum_{l} \varrho_{m}\left(C_{2}, \ldots, C_{m}, \tilde{C}_{l} \mid \Lambda\right) .
\end{aligned}
$$

Calling now $\overline{\mathscr{M}}\left(\tilde{\Theta}\left(C_{1}\right)\right)$ the set of all configurations within $\tilde{\Theta}\left(C_{1}\right)$ giving rise to sets of chains non "intersecting" $C_{1}$ and calling $\bar{\Xi}\left(\tilde{\Theta}\left(C_{1}\right)\right)$ the 
associated grand partition function, we may write:

$\varrho_{C_{1}}\left(C_{1}, \ldots, C_{m} \mid \Lambda\right)=\frac{\bar{\Xi}\left(\tilde{\Theta}\left(C_{1}\right)\right) \sum_{\left\{C_{1}^{0}, \ldots, C_{s}^{0}\right\}}^{\sum_{2}^{\prime}} \prod_{i}^{s} \frac{\Xi\left(\tilde{\Theta}\left(C_{i}^{0}\right)\right)}{\Xi(\Lambda)} \Xi_{0}\left(\tilde{\Theta}\left(C_{i}^{0}\right)\right)}{\Xi_{0}}$

where the meaning of the primed sum is the same as in Section I. In addition, noting that Eq. (3) can be written ás:

$\varrho_{m}\left(C_{1}, \ldots, C_{m} \mid \Lambda\right)=\frac{\Xi\left(\tilde{\Theta}\left(C_{1}\right)\right) \sum_{\left\{C_{1}^{0} \ldots, C_{s\}}^{0}\right\}}^{\sum_{1}^{\prime}} \prod_{i}^{s} \frac{\Xi\left(\tilde{\Theta}\left(C_{1}^{0}\right)\right)}{\Xi(\Lambda)} \Xi_{0}(\Lambda)}{\Xi_{0}\left(\tilde{\Theta}\left(C_{i}^{0}\right)\right)}$

comparing (A.2) to (A.3), we get:

$$
\varrho_{m}\left(C_{1}, \ldots, C_{m} \mid \Lambda\right)=\lambda\left(C_{1}\right) \varrho_{C_{1}}\left(C_{2}, \ldots, C_{m} \mid \Lambda\right)
$$

where we have set

$$
\lambda\left(C_{1}\right)=\frac{\Xi\left(\tilde{\Theta}\left(C_{1}\right)\right)}{\bar{\Xi}\left(\tilde{\Theta}\left(C_{1}\right)\right)} .
$$

Finally, the relation (A.4) together with (A.1) gives Eqs. (4) and (5), as required.

\section{Appendix B}

Lemma. For $z_{A}=z_{B}=z$ and $z$ high enough, we have the bounds:

$$
\begin{aligned}
& \varrho_{m}\left(C_{1}, \ldots, C_{m}\right) \leqq e^{-\alpha \sum_{1}^{n}\left|C_{1}\right|} \\
& \varrho_{1}\left(C_{1}\right) \leqq \lambda\left(C_{1}\right) \leqq e^{-\alpha\left|C_{1}\right|}
\end{aligned}
$$

where $\alpha=\frac{z R^{2}}{72}$, and $|C|$ is the length of the perimeter of the chain $C$.

Proof. The proof is substantially contained in Ruelle's paper [5], but to avoid ambiguities, that could arise from the different notations, we will rephrase it.

Any configuration $Y$ of $A$ and $B$ particles, belonging to $\mathscr{M}(\tilde{\Theta}(C))$, is associated to a collection of contours containing the outer ones $\gamma_{1}, \ldots, \gamma_{n}$, that make $C$, and a set of contours, $\gamma_{1}^{\prime}, \ldots, \gamma_{k}^{\prime}$, internal to $\Theta(C)$.

We write this collection as the union of disjoint pieces $\Gamma_{C}^{0}, \Gamma_{C}^{1}, \ldots, \Gamma_{C}^{l}$. Where each piece is a smallest subset of contours such that, if two contours have a distance less then $\frac{8 R}{3 \sqrt{2}}$ they belong to the same piece. 
Calling $\Gamma_{c}^{0}$ the piece containing the external contours, we say that a point $x$ is interior to $\Gamma_{C}^{0}$ if a path, coming from infinity, crosses $\Gamma_{C}^{0}$ an odd number of times before reaching $x$ (see Fig. 1).

From $Y$ we obtain a class $Y^{*}$ of configurations as follows:

a) All $A$-particles interior to $\Gamma_{C}^{0}$ are changed to $B$-particles and viceversa.

b) Let $G\left(\Gamma_{C}^{0}\right)$ be a band consisting of all the little squares which have one side or corner touching the contours belonging to $\Gamma_{C}^{0}$; $A$-particles are placed in an arbitrary manner in $G\left(\Gamma_{c}^{0}\right)$.

If we call $\mathscr{M}^{*}(\tilde{\Theta}(C))$ the collection of all configurations, obtained via the previously described transformation from $\mathscr{M}(\tilde{\Theta}(C))$, the following inequalities hold:

$$
\varrho_{m}\left(C_{1}, \ldots, C_{m}\right) \leqq \prod_{1}^{m} \frac{\Xi\left(\tilde{\Theta}\left(C_{i}\right)\right)}{\Xi^{*}\left(\tilde{\Theta}\left(C_{i}\right)\right)} \quad(m=1,2, \ldots) .
$$

Calling now $\mathscr{M}\left(\hat{\Theta}(C), \Gamma_{C}^{0}\right)$ the subset of the configurations belonging to $\mathscr{M}(\tilde{\Theta}(C))$ that have $\Gamma_{C}^{0}$ among their disjoint pieces and $\Xi\left(\tilde{\Theta}(C), \Gamma_{C}^{0}\right)$ the associated grand partition sum, we get

$$
\Xi(\Theta(C))=\sum_{\Gamma_{C}^{0}} \Xi\left(\tilde{\Theta}(C), \Gamma_{C}^{0}\right)
$$

where the sum is over all possible $\Gamma_{C}^{0}$.

Then, noting that when $z_{A}=z_{B}$

$$
\Xi^{*}\left(\tilde{\Theta}(C), \Gamma_{C}^{0}\right)=\Xi\left(\tilde{\Theta}(C), \Gamma_{C}^{0}\right) \Xi\left(G\left(\Gamma_{C}^{0}\right)\right),
$$

where $\Xi\left(G\left(\Gamma_{C}^{0}\right)\right)$ is the grand partition function for $A$-particles in $G\left(\Gamma_{C}^{0}\right)$, and, as in Ruelle [5], with $\alpha=\frac{z R^{2}}{72}$

$$
\Xi\left(G\left(\Gamma_{C}^{0}\right)\right) \geqq e^{2 \alpha|C|}
$$

we get, using standard estimates,

$$
\begin{aligned}
\frac{\Xi(\tilde{\Theta}(C))}{\Xi^{*}(\tilde{\Theta}(C))} & \leqq \sum_{\Gamma_{C}^{0}} \frac{1}{\Xi\left(G\left(\Gamma_{C}^{0}\right)\right)} \\
& \leqq e^{-2 \alpha|C|} \sum_{0}^{\infty} \frac{1}{m !}|C| \sum_{12}^{\infty}\left(3^{\left(\frac{\mathscr{O}}{6}+1\right)} e^{-2 \alpha)} \leqq e^{-\alpha|C|}\right.
\end{aligned}
$$

when $\alpha>\frac{1}{8}$.

The inequality for $\lambda\left(C_{1}\right)$ is obtained by the same arguments observing that $\mathscr{M}(\tilde{\Theta}(C)) \supset \mathscr{M}^{*}(\tilde{\Theta}(C))$. 


\title{
References
}

1. Minlos, R.A., Sinai, J.C.: Transl. Math. USSR-Sbornik 2, 335 (1967)

2. Minlos, R.A., Sinai, J.C.: Transl. Moscow Math. Soc. 19, 121 (1968)

3. Peierls, R.: Proc. Cambridge Phil. Soc. 32, 477 (1936)

4. Widom, B., Rowlinson, J.S.: J. Chem. Phys. 52, 1670 (1970)

5. Ruelle,D.: Phys. Rev. Letters 27, 1040 (1971)

6. Ruelle,D.: Ann. Phys. 25, 109 (1963)

7. Minlos, R.A., Sinai, J.C.: Transl. Moscow Math. Soc. 17, 237 (1967)

8. Capocaccia,D., Cassandro, M., Ciccotti, G.: Commun. math. Phys. 29, 31 (1973)

9. Cassandro,M., Gallavotti, G., Lebowitz,J.L., Monroe,J.L.: Commun. math. Phys. 32, 153 (1973)

10. Martin-Löf,A.: Commun. math. Phys. 32, 75 (1973)

11. Da Fano, A.: Rome University thesis, Physics Institute, July 1973 (unpublished)

12. Feller, W.: Introduction to Probability theory and its applications, Pag. 86, Vol. $I^{\circ}$. New York: Wiley \& Sons 1950

Communicated by G. Gallavotti

\author{
M. Cassandro \\ A. Da Fano \\ Istituto di Fisica Teorica \\ Università di Roma \\ Piazzale delle Scienze \\ 00185 Roma, Italy
}

